\title{
Open prostatectomy: the safety and efficacy of this procedure in developing countries
}

\begin{abstract}
Objective: To propose the efficacy and safety of open suprapubic transvesical prostatectomy. Benign prostate hypertrophy (BPH) affects the majority of men in their life after the age of 40 years and is the most common cause of lower urinary tract symptoms (LUTS). Despite the popularity of transurethral resection of the prostate (TURP), open prostatectomy remains suitable surgical options for the management in patients with benign prostate hypertrophy living in developing countries due to scarcities of endoscopic tools.
\end{abstract}

Method: In May 2018 to January 2020, 24 cases of benign prostate hypertrophy underwent suprapubic transvesical open prostatectomy at Mogadishu Somali Turkey Reccep Tayyip Erdogan Training and Research Hospital. The outcome of these cases was prospectively analyzed. All patients with a suspect of prostate cancer, and abnormal coagulation profile and patients with a high-risk group (high-risk comorbidities) were excluded from the study.

Result: The mean age of patients was 70.5 years with an age range of 50-90. The average prostate size was $146.5 \mathrm{gr}$. Acute urinary retention was the predominant presentation followed by nocturia and urgency. The mean preoperative hemoglobin of the patients was $13.3 \pm 1.8 \mathrm{mg} / / \mathrm{dl}$. The average intraoperative bleeding was $550 \mathrm{ml}$ and the mean postoperative hemoglobin was $10.6 \pm 1.6 \mathrm{mg} / \mathrm{dl}$. The commonest postoperative complication was clot retention and the predominant concomitant condition was bladder stones. The mean hospital stay was $3.4 \pm 1.5$ days.

Conclusion: Open prostatectomy is safe and effective operative management for benign prostate hyperplasia in developing countries due to scarcities of endoscopic equipment and larger sizes of the prostate gland on presentation.

Keywords: prostate, open prostatectomy, clot, acute urinary retention, bleeding, nocturia
Volume 8 Issue 2 - 2020

\author{
Abdikarim Hussein Mohamed, Hussein Ali \\ Mohamud \\ Department of urology, Mogadishu Somali Turkish Training and \\ Research Hospital, Somalia \\ Correspondence: Abdikarim Hussein Mohamed, Department \\ of urology, Mogadishu Somali Turkish Training and Research \\ Hospital, Mogadishu, Somalia, Tel 00252615167/82, \\ Email abdikarimgabeyre@gmail.com
}

Received: April 09, 2020 | Published: April 28, 2020
Abbreviations: LUTS, lower urinary tract symptoms; $\mathrm{BPH}$, benign prostate hypertrophy; TURP, transurethral resection of the prostate; TPSA, total prostate-specific; DRE, digital rectal examination

\section{Introduction}

Transurethral resection of the prostate (TURP) is the gold standard surgical management of benign prostate hyperplasia and became more popular over the world. Despite this, open prostatectomy still offers the benefit of lower failure rates, less risk of TURP syndrome compared with TURP. Due to the scarcities of endoscopic equipment in developing countries, open prostatectomy may be safely performed in this era while simple suprapubic transvesical open prostatectomy is the preferred approach. ${ }^{1,2}$ Age is the most common risk factorassociated with benign prostate hyperplasia (BPH) and the incidence of BPH rises with age. The estimated prevalence is $25 \%$ in men aged 40 to 79 years. ${ }^{3}$ Benign prostate hyperplasia characterized by symptoms that disrupt the patient's normal urination, sexual function, and quality of life. ${ }^{4,5}$ The aim of our study is to propose the efficacy and safety of open suprapubic transvesical prostatectomy in developing countries.

\section{Methods}

In May 2018 to January 2020, 24 cases ofbenign prostate hypertrophy presenting with either acute urinary retention orlower urinary tract symptoms due to bladder neck obstruction underwent suprapubic transvesical open prostatectomy at Mogadishu Somali Turkey Reccep Tayyip Erdogan Training and Research Hospital. All routine preoperative investigations were made including complete blood count, metabolic panel, total prostate-specific antigen (TPSA), urine analysis, and abdominal ultrasound. All patients with a suspect of prostate cancer (high TPSA, abnormalities of digital rectal examination), abnormal coagulation profile and patients with a highrisk group (high-risk comorbidities) were excluded from the study. Approval form was obtained from the ethics committee of Mogadishu Somali Turkey Reccep Tayyip Erdogan Training and Research Hospital and all patients were taken informed consent.

\section{Procedure}

Spinal anesthesia or general anesthesia was used. The patient was positioned on a supine position, after preparing and draping under the sterile procedure, a urethral catheter has inserted the patient with 200$300 \mathrm{ml}$ saline into the bladder. Midline vertical incision about $10 \mathrm{~cm}$ or Pfannenstiel incision was made. The bladder was gained after dissection of the rectus abdominus muscle laterally and exposing the space of retzius. After cystostomy, the bladder was better visualized and cauterization of median lobe borders was performed to protect the ureteral orifices and to prevent unwanted sequels; blunt digital enucleation was performed and 5 and 7 O-clock hemostatic 2-0 Vicryl absorbable sutures and other visible bleeding sites were performed. Three-way urethral catheter inflating 20-40 cc and cystostomy catheter when needed was placedon the patient. The irrigation channel of the three-way catheter was connected to the normal saline for irrigation until the urine becomes clear mostly for 24 hours. The bladder was closed in double-layer fashion using 2-0 vicryl absorbable sutures. The drain was placed in the space of retzius. Standard traction was 
used as needed. None of the patients need a blood transfusion in the perioperative period. SPSS version 23 was used for analyzes of parameters.

\section{Results}

A total of 24 cases of benign prostate hypertrophy underwent suprapubic transvesical open prostatectomy at Mogadishu Somali Turkey Reccep Tayyip Erdogan Training and Research Hospital. All patients take a single dose of cephazolininjection 1 gram with 1 hour of operation. The mean age of patients was 70.5 years with an age range of 50-90. The average prostate size was $146.5 \mathrm{gr}$. Four patients needed a preoperative blood transfusion $(16.7 \%)$. Acute urinary retention was the predominant presentation followed by nocturia and urgency. The mean preoperative serum creatinine was $1.26 \mathrm{mg} / \mathrm{dl}$. Sixpatients had renal failure and 3 patients had hydronephrosis on ultrasound preoperatively. The mean preoperative hemoglobin of the patients was $13.3 \pm 1.8 \mathrm{mg} / / \mathrm{dl}$. The average intraoperative bleeding was $550 \mathrm{ml}$ and the mean postoperative hemoglobin was $10.6 \pm 1.6 \mathrm{mg} / \mathrm{dl}$ (Table 1). Two patients needed blood transfusion postoperatively $(8.3 \%)$. Clot retention was the most common postoperative complication encountered in 5 patients $(20.8 \%)$. Most patients with clot retention postoperatively were managed with intermittent manual irrigation; one patient with clot retention needed cystoscopic evacuation (Table 2 ). One patient developed surgical site infection and were treated with systemic antibiotics after culture results (4.1\%). One patient developed fascial dehiscence needing secondary suture. Bladder stoneswere the most common concomitant condition associated with benign prostate hyperplasia in our study and there were 7 patients ( $29 \%$ of the cases) having concomitants of bladder stones. The mean hospital stay was $3.4 \pm 1.5$ days and catheterization time was 7days. Two patients had prostate cancer found after histopathology examination $(8.3 \%)$. Diabetes was the most common comorbidities encountered in three patients (12.5\% of the cases), $8.3 \%$ of the cases (two patients) having hypertension, one patient having heart failure, 1 patient having Chronic Obstructive Pulmonary Disease (Table 3).

Table I Preoperative, intraoperative and postoperative average hemoglobin loss

\begin{tabular}{|c|c|}
\hline Perioperative hemoglobin & $\begin{array}{l}\text { Hemoglobin levels and } \\
\text { average changes }\end{array}$ \\
\hline Preoperative average hemoglobin & $13.3 \pm 1.8 \mathrm{mg} / / \mathrm{dl}$ \\
\hline Postoperative average hemoglobin & $10.6 \pm 1.6 \mathrm{mg} / \mathrm{dl}$ \\
\hline Intraoperative average hemoglobin loss & $2.7 \mathrm{mg} / / \mathrm{dl}$ \\
\hline Intraoperative average bleeding & $550 \mathrm{ml}$ \\
\hline
\end{tabular}

Table 2 Occurrence of clot retention

\begin{tabular}{lll}
\hline Clot episodes & Number of patients & Percentage \\
\hline Yes & 5 & $20.80 \%$ \\
No & 19 & $79.20 \%$ \\
\hline
\end{tabular}

Table 3 Patient's comorbidities

\begin{tabular}{lll}
\hline Comorbidities & $\begin{array}{l}\text { Number of } \\
\text { patients }\end{array}$ & Percentage \\
\hline Renal failure & 6 & $25 \%$ \\
Diabetes & 3 & $12.50 \%$ \\
$\begin{array}{l}\text { Hypertension } \\
\text { Chronic Obstructive }\end{array}$ & 2 & $8.30 \%$ \\
$\begin{array}{l}\text { Pulmonary Disease } \\
\text { Heart failure }\end{array}$ & 1 & $4 \%$ \\
\hline
\end{tabular}

\section{Discussion}

There is no available data regarding the prevalence of $\mathrm{BPH}$ in Somalia. Most of the Somali people live in rural areas living with some financial problems that lead less access for medical and surgical management of BPH and this reason,the majority of these men do not seek medical care until they develop bladder outlet obstruction related sequels including acute urinary retention, recurrent/chronic urinary tract infections, bladder stones and bladder diverticula, hydronephrosis, and renal insufficiency. The average prostate weight in our study is 146.5 gram and larger than all other reported studies in the literature. In a study from Seretta $\mathrm{V}$ presented an average prostate weight of 112.3gram. ${ }^{6}$ In another study from Tubaro A et al. ${ }^{8}$ reported an average prostate weight of 63 gram. $^{7}$ N.A. Berhanu reported an average prostate weight of $73.6 \mathrm{gram}$ in their study. ${ }^{8}$ The indications of open prostatectomy in our series were larger sizes of prostate gland, concomitant bladder stones or bladder diverticula, and the scarcities of available endoscopic equipment and patients not willing reoperations. ${ }^{7,9}$

Suprapubic transvesical prostatectomy, simple retropubic prostatectomy, and perineal prostatectomy are the three operative techniques for open simple prostatectomy. A substantial finding noted in our study is that the greatest advantage of the suprapubic transvesical open prostatectomy approach over the other two approaches is that it is suitable for synchronized management of other concomitants including bladder stone or bladder diverticula. Also it is a better choice for larger median lobes that protrude in the bladder and better visualization of ureteral orifices to prevent unwanted events that can lead obstruction of the ureteral orifices and secondary renal failure. TURP is the gold standard for the management of BPH and became popular over the world. One significant finding confirmed in our study is that open prostatectomy has advantages over transurethral prostate resection including fewer rates of reoperation, lower mortality rate, not associated with TURP syndrome and lower health-related costs. ${ }^{10}$ Open prostatectomy is superior over TURP for symptomatic relief and improvement of urination, but it is an invasive procedure and high morbidity compared to transurethral prostate resection. ${ }^{11}$

Complete medical history, physical examination including the digital rectal examination (DRE), laboratory investigations including Total Prostate Specific Antigen (TPSA), renal function tests, and urine analysis and ultrasound are obligatory work-up for the diagnosis of BPH as coincided by the World Health Organization (WHO) sponsoredInternational consensus committee (ICC), UK British prostate group and American guideline group. ${ }^{8}$ Suspect or presence for bladder cancer and/or prostate cancer is contraindicated of open 
simple prostatectomy. Complete enucleation of the prostate adenoma was achieved by Fuller before Fuller, McGill, and Belfield in the 1800 s performed for partial enucleation of prostate adenoma. Lower and Harris proposed the use of hemostatic sutures ${ }^{12,13}$ and modified by Hryntschak.

Hemostatic 2:0 Vicryl absorbable sutures at 5 and 7 O-clock and other visible bleeding sites were performed for the patients of the current study and it is associated with lower bleeding rates and lower mortality rates.As reported in the literature and noted in our study, closing of the bladder in double-layer fashion with 2-0 absorbable vicryl suture may prevent urine leak in most of the patients.Dela Pena presented the use of removable purse-string suture in 1962 to decrease bleeding associated complications. ${ }^{14}$ Our study advocate the use of continuous bladder irrigation via urethral catheter until the urine became clear up to 24-48 hours whilesome studies in the literature support the use of suprapubic catheter. ${ }^{14}$ Clot retention was the most common postoperative complication in our study encountered in five patients $(20.8 \%)$. Most patients with clot retention postoperatively were managed with intermittent manual irrigation; one patient with clot retention needed cystoscopic evacuation. Some studies support removing the urethral catheter firstly after three days postoperatively and the cystostomy clamped and removed after that and then drain removed lastly. Cystostomy catheter when used was removed by the second day postoperatively in this study. Following the next days, drain was removed. On day seven, the urethral catheter was removed. There was no significant morbidity associated with this method compared to the above mentioned method.

Meier et al. ${ }^{15}$ reported the findings of their studywith the comparison for their study with a study series of TUR-P reported by Mebustet al. Meier's studywas related lower early complication rate including clot retention, intraoperative bleeding, need for blood transfusion and other early complications and accounted about $19 \%$ and mortality rate of $0 \%$ compared with Mebust's study ranging about $25 \%$ and $02 \%$ respectively.Clot retention is common in the open prostatectomy group compared to the TURP group. ${ }^{15}$ Besides these studies, we achieved lower complication rate compared to these studies andaccomplisheda $0 \%$ mortality rate as the goal is to reach no mortality associated with open prostatectomy. Condie et al. ${ }^{10}$ reported in their study a $1 \%$ blood transfusion ratewhileKadirCeylan reported a $10 \%$ blood transfusion rate. In our series, $8.3 \%$ of the cases needed blood transfusion postoperatively which is lower the study from KadirCeylan. ${ }^{10], 11}$ The mean hospital stay was $3.4 \pm 1.5$ days. Most of the patients were discharged home with a urethral catheter by day 3 or 4 postoperatively.

\section{Conclusion}

Open prostatectomy is safe and effective operative management for benign prostate hyperplasia in developing countries due to scarcities of endoscopic equipment and larger sizes of prostate gland on presentation.

\section{Acknowledgments}

None.

\section{Conflicts of interest}

The authors declare no conflict of interest and this study received no financial support.

\section{References}

1. Holtgrewe HL, Mebust WK, Dowd JB, et al. Transurethral prostatectomy: practice aspects of the dominant operation in American urology. J Urol. 1989;141(2):248-253.

2. Roos NP, Wennberg JE, Malenka DJ, et al. Mortality and reoperation after open and transurethral resection of a prostate for benign prostatic hyperplasia. N Engl J Med. 1989;320(17):1120-1124.

3. Berry SJ, Coffey DS, Walsh PC, et al. The development of human benign prostatic hyperplasia with age. J Urol. 1984;132(3):474-479.

4. Rosen RC, Giuliano F, Carson CC. Sexual dysfunction and lower urinary tract symptoms (LUTS) associated with benign prostatic hyperplasia (BPH). Eur Urol. 2005;47(6):824-37.

5. Nickel JC, Elhilali M, Vallancien G. Benign prostatic hyperplasia $(\mathrm{BPH})$ and prostatitis: Prevalence of painful ejaculation in men with clinical BPH. BJU Int. 2005;95(4):571-574.

6. Serretta V, Morgia G, Fondacaro L, et al. Open prostatectomy for benign prostatic enlargement in southern Europe in the late 1990s: A contemporary series of 1800 interventions. Urology. 2002;60(4):623627 .

7. Tubaro A, Carter S, Hind A, et al. A prospective study of the safety and efficacy of suprapubic transvesical prostatectomy in patients with benign prostatic hyperplasia. J Urol. 2001;166(1):172-176.

8. NA Berhanu. The Safety and Efficacy of Trans-Vesical Prostatectomy Done at a Primary General Hospital Setting in Ethiopia. East and Central African Journal of Surgery. 2008;13(2):1-2.

9. Servadio C. Is open prostatectomy really obsolete? Urology. 1992;40(19):419-421.

10. Condie JD, Cutherell L, Mian A. Suprapubic prostatectomy for benign prostatic hyperplasia in rural Asia: 200 consecutive cases. Urology. 1999;54(6):1012-1016.

11. Ceylan K. Open prostatectomy: The results of a series of 320 cases in rural area. Eur J Gen Med. 2006;3(1):11-15.

12. WE Lower. Complete closure of the bladder following prostatectomy. JAMA. 1927;89:749-751.

13. S Harry Harris, Ch M Sydney. Suprapubic prostatectomy with closure. Surg Gynecol Obstet. 1930;50:251-260.

14. Cohen SP, Kopilnick MD, Robbins MA. Removable purse-string suture of the vesical neck during suprapubic prostectomy. $J$ Urol. 1969;102(6):720-722.

15. Meier DE, Tarpley JL, Imediegwu OO, et al. The outcome of suprapubic prostatectomy: A contemporary series in the developing world. Urology. 1995;46(1):40-44.

16. Mebust WK, Holtgrewe HL, Cockett ATK, et al. Transurethral prostatectomy: Immediate and postoperative complications. A cooperative study of 13 participating institutions evaluating 3,885 patients. J Urol. 1989;141(2):243-247. 